\title{
Cyclosporin A therapy in refractory non-infectious childhood uveitis
}

\author{
D J Kilmartin, J V Forrester, A D Dick
}

\begin{abstract}
Aims-To assess the immunosuppressive efficacy, steroid sparing effect and adverse effects of cyclosporin A (CsA) therapy in refractory non-infectious childhood uveitis.

Methods-A retrospective case series review of the medical records of children on CsA therapy attending a tertiary referral centre for refractory endogenous uveitis was performed. Low dose $(\leqslant 5.0 \mathrm{mg} / \mathrm{kg} /$ day) CsA therapy was started either as monotherapy or in combination with other agents. The CsA immunosuppressive efficacy was assessed by visual acuity and binocular indirect ophthalmoscopy (BIO) score outcomes and steroid sparing effect by growth charts and ability to withdraw or maintain a low steroid dose. Possible CsA adverse effects were monitored by routine biochemistry (including serum creatinine) and haematological tests, blood pressure recordings, and symptoms.
\end{abstract}

Results-14 patients (25 eyes, 10 males, four females) were recruited with steroid failure as the most common CsA indication. Age (mean (SD)) at start of CsA therapy was $8.7(4.1)$ years with a duration of CsA therapy of 20.9 (range 3.5-88.3) months at a maintenance CsA dose of 4.0 (1.0) $\mathrm{mg} / \mathrm{kg} / \mathrm{day}$. From baseline, visual acuity improved or was maintained in 23 $(92 \%)$ eyes and BIO score improved in 19 (76\%) eyes. Height centiles were preserved and the maintenance prednisolone dose was $6.3(3.3) \mathrm{mg} / \mathrm{day}$, where required, in 10 (71\%) patients. Nephrotoxicity was not observed, with transient systemic hypertension developing in one patient. Minor adverse effects were more common but were well tolerated.

Conclusions-Cyclosporin A therapy is effective and safe in the medium term, if closely monitored, in refractory noninfectious childhood uveitis.

(Br f Ophthalmol 1998;82:737-742)

University of Aberdeen

Medical School,

Aberdeen

D J Kilmartin

J V Forrester

A D Dick

Correspondence to: Mr D J Kilmartin,

Department of

Ophthalmology, University

of Aberdeen Medical School,

Foresterhill, Aberdeen AB25 2ZD.

Accepted for publication 29 January 1998
$5.2 \%$ and $10.6 \%$. Presentat recent study reporting $26 \%$ of eyes having visual acuity of less than 20/200 at first referral, ${ }^{1}$ possibly due to asymptomatic insidious onset or uveitis in a preverbal child. Corticosteroids have traditionally formed the mainstay of systemic immunosuppression in childhood uveitis. ${ }^{19-11}$ However, the well described adverse effects of systemic corticosteroids on the immature immune, metabolic, and skeletal systems, ${ }^{12-18}$ which may result in permanent disability including growth retardation, ${ }^{15-18}$ restrict their long term use in children. Although alternative immunosuppressive agents, such as cyclosporin A (CsA), antimetabolites, and alkylating agents, when carefully administered and monitored, have relatively few permanent adverse effects in the therapy of adult ocular inflammatory disease, ${ }^{12}{ }^{19-24}$ there are additional concerns which restrict their use in childhood uveitis. These include the potential long term risks of neoplasia, myelosuppression, nephrotoxicity, or hypertension. ${ }^{12} 222627$ It has therefore, not surprisingly been reported that the potential benefit of cytotoxic agents in the treatment of intractable childhood uveitis is outweighed by the risk of serious adverse effects. ${ }^{11}$

Cyclosporin A is a powerful steroid sparing immunosuppressive agent which has been shown to be both effective and relatively safe in low doses in the treatment of refractory endogenous posterior uveitis in adults. ${ }^{20-24}$ There are, however, few reports which examine the efficacy of CsA therapy in refractory noninfectious childhood uveitis. ${ }^{12829}$ One of the main concerns with CsA therapy is nephropathy but a large review in patients treated for a range of autoimmune diseases found CsA less nephrotoxic in children compared with adults, ${ }^{27}$ possibly because of greater CsA clearance in children. ${ }^{30}$ The maximal degree of CsA induced renal dysfunction can be measured by the percentage increase in serum creatinine above the patient's baseline value which is reported to be the best predictor of CsA induced nephropathy. ${ }^{27}$ To minimise dose dependent CsA induced nephropathy, many reports propose maintaining a CsA dose of 5 $\mathrm{mg} / \mathrm{kg}$ per day or less and then titrating the dose to keep within a $30 \%$ increase in serum creatinine over the baseline value $\mathrm{e}^{27} 3132$

The efficacy of CsA as a steroid sparing immunosuppressant is highlighted within other branches of paediatric medicine where growth is preserved after renal $^{180^{33-36}}$ and cardiac $^{37}$ transplantation in children, controlling nephrotic syndrome, ${ }^{3839}$ and treating recent onset type I insulin dependent diabetes mellitus. ${ }^{40}$ Therefore, the aims of this study were to examine the immunosuppressive efficacy, steroid sparing effect, and adverse effects 
Table 1 Patient diagnosis and duration of cyclosporin A therapy

\begin{tabular}{llll}
\hline $\begin{array}{l}\text { Patient No/sex/age } \\
\text { at onset of uveitis } \\
\text { (years) }\end{array}$ & Diagnosis & $\begin{array}{l}\text { Classification } \\
\left(I U S G^{\star} \text { )/uni/bilaterality }\right.\end{array}$ & $\begin{array}{l}\text { Duration CsA } \\
\text { therapy (months) }\end{array}$ \\
\hline 1/F/3.9 & JCA & Panuveitis/both eyes & 88.3 \\
$2 / \mathrm{M} / 9.2$ & Pars planitis & Intermediate uveitis/both eyes & 52.2 \\
3/M/3.9 & Idiopathic & Intermediate uveitis/both eyes & 28.3 \\
4/M/3.8 & Sympathetic ophthalmia & Posterior uveitis/both eyes & 25.0 \\
$5 / \mathrm{M} / 5.8$ & Pars planitis & Intermediate uveitis/both eyes & 24.9 \\
$6 / \mathrm{M} / 6.7$ & Sympathetic ophthalmia & Posterior uveitis/both eyes & 14.3 \\
$7 / \mathrm{M} / 3.0$ & Idiopathic & Intermediate uveitis/both eyes & 14.1 \\
$8 / \mathrm{M} / 5.6$ & Pars planitis & Intermediate uveitis/both eyes & 11.7 \\
$9 / \mathrm{F} / 2.3$ & JCA & Panuveitis/both eyes & 10.6 \\
$10 / \mathrm{F} / 11.4$ & JCA & Intermediate uveitis/right eye & 6.5 \\
$11 / \mathrm{M} / 8.6$ & Idiopathic & Panuveitis/right eye & 5.1 \\
$12 / \mathrm{M} / 9.3$ & Pars planitis & Intermediate uveitis/both eyes & 4.5 \\
$13 / \mathrm{F} / 6.0$ & Idiopathic & Posterior uveitis/right eye & 3.8 \\
$14 / \mathrm{M} / 15.2$ & Pars planitis & Intermediate uveitis/both eyes & 3.5 \\
\hline
\end{tabular}

*IUSG = International Uveitis Study Group; CsA = cyclosporin A; JCA = juvenile chronic arthritis.

of CsA therapy in refractory endogenous childhood uveitis.

\section{Methods}

PATIENTS AND CLINICAL MONITORING

From the uveitis database of patients attending the uveitis clinics at Aberdeen Royal Hospitals NHS Trust (ARHT), a tertiary referral centre, a consecutive series was collected and medical records reviewed of patients under the age of 16 years who received systemic CsA for refractory non-infectious uveitis. Patients received CsA monotherapy or in combination with systemic low dose steroids and/or other immunosuppressives. Previous history and follow up data were collected from the referring physician when indicated. Clinical data collected included age, uveitis diagnosis, uni/bilaterality of uveitis, sex, prior/current periocular or systemic steroid treatment including dosage and duration, indication for CsA treatment, CsA dosage variables, duration of therapy, and adverse effects. Uveitis was classified both anatomically, following the International Uveitis Study Group classification system, ${ }^{41}$ and also by systemic disease associations or as isolated defined uveitis entities, such as pars planitis or sympathetic ophthalmia. Infectious aetiology was excluded clinically and/or by associated negative serology for Toxoplasma, Toxocara, herpes simplex and zoster viruses, cytomegalovirus, Epstein-Barr virus, Treponema, Borrelia, and negative skin tuberculin test when indicated. Visual function was assessed by recording the best corrected visual acuity (BCVA), using Kay's pictures, Sheridan-Gardiner test types, or the Snellen chart (depending on the age and comprehension of the patient), before CsA therapy and at the last clinic visit. Amblyopia was deemed to contribute to

Table 2 Indications for cyclosporin A therapy

\begin{tabular}{lll}
\hline Indication & $\begin{array}{l}\text { No of } \\
\text { patients }\end{array}$ & Percentage* \\
\hline $\begin{array}{l}\text { Failure of systemic steroids/ } \\
\text { persistent severe IOI }\end{array}$ & 6 & 43 \\
$\begin{array}{l}\text { Chronic systemic steroid sparing } \\
\quad \text { therapy }\end{array}$ & 6 & 43 \\
$\begin{array}{l}\text { Toxicity of systemic steroids } \\
\text { Specific CsA therapy indication }\end{array}$ & 2 & 21 \\
\end{tabular}

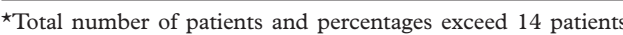
and $100 \%$ respectively as some patients had more than one indication. IOI = intraocular inflammation; $\mathrm{CsA}=$ cyclosporin $\mathrm{A}$. reduced final BCVA when age at first presentation was under 8 years. The anti-inflammatory efficacy of CsA therapy was assessed by measuring the binocular indirect ophthalmoscopy (BIO) score. $^{42}$ All patients were examined at each visit by the same ophthalmologist (JVF) to achieve standardisation. All adverse effects related to prior high dose and/or chronic systemic steroid therapy, such as cushingoid features and growth retardation were recorded. Heights and weights were recorded on age and sex matched charts ${ }^{43}$ for the duration of therapy where data were available. Similarly, adverse effects of CsA therapy, especially renal dysfunction and systemic hypertension, were closely monitored. Renal function was assessed by baseline isotope glomerular filtration rate, and in some cases by baseline creatinine/ lithium clearance, ${ }^{44}$ with serial assessments of serum creatinine at each follow up visit. The full blood count, electrolytes including magnesium, blood glucose, total cholesterol levels, serum uric acid, and CsA levels were measured at each visit.

INDICATIONS FOR AND OPTIMISING CSA THERAPY Indications for starting low dose CsA therapy included one or more of the following: (1) steroid dependency; (2) toxicity from previous steroid/immunosuppressive therapy; (3) a specific indication for CsA therapy (such as sympathetic ophthalmia); (4) failure/ inadequate response with steroid/other immunosuppressive therapy. Contraindications to CsA therapy included abnormal baseline renal/ liver function tests or uncontrolled systemic hypertension. Detailed informed consent, involving an explanation of the potential risks and benefits of CsA therapy, was obtained from both patient, if possible, and parents. Cyclosporin A therapy was commenced at 5.0 $\mathrm{mg} / \mathrm{kg}$ daily in two divided doses. Follow up outpatient assessments occurred at 2 weeks, 1 month, and then on a monthly basis for the first year with subsequent visits every 2-3 months or sooner if clinically indicated. Subsequent CsA dosage adjustments and/or the addition of other steroid sparing agents or steroid dosage adjustments, as in other reports, ${ }^{21}{ }^{245-47}$ were titrated according to the degree of vitreous and retinal inflammation, rather than the visual acuity, aiming to achieve the lowest possible dosage that maintained adequate inflammatory control yet minimised adverse effects. If an inadequate response to CsA therapy at the maximal dose occurred, azathioprine $(1.5-2.0 \mathrm{mg} / \mathrm{kg}$ daily) was added to the regimen. If an insufficient antiinflammatory response persisted, the CsA dose was increased to slightly exceed the usual upper limit of $5 \mathrm{mg} / \mathrm{kg}$ daily in order to achieve adequate immunosuppression. If intraocular inflammation was clinically controlled, a slow reduction of CsA dosage was attempted. Any subsequent recurrences or rebound increase in intraocular inflammation during steroid or CsA dose reduction was managed by a compensatory increase in steroid and/or CsA dosage. Long term remission of intraocular inflammation was confirmed when CsA could 


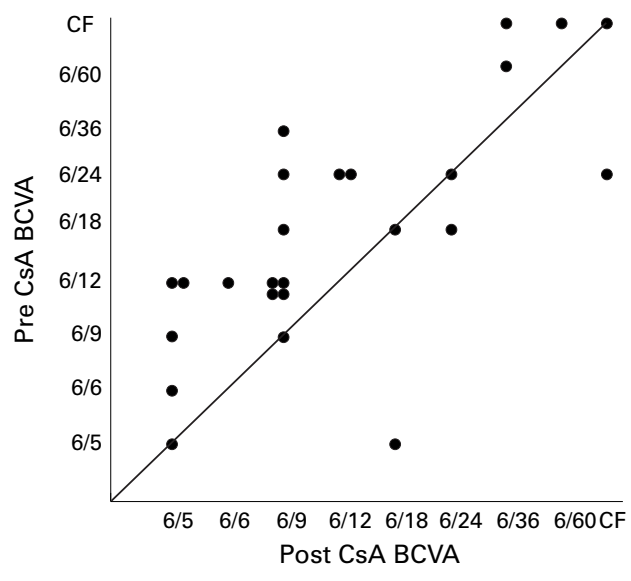

Figure 1 Best corrected visual acuity (BCVA) outcome on cyclosporin $A$ (Cs $A$ ) therapy. Affected eyes $n=25$.

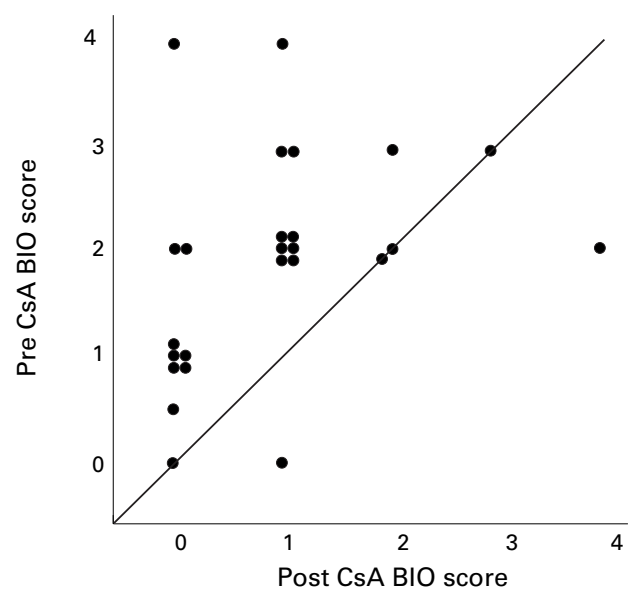

Figure 2 Binocular indirect ophthalmoscopy (BIO) outcome on cyclosporin $A$ (Cs $A$ ) therapy. Affected eyes $n=25$.

be successfully withdrawn for at least 6 months or if patients received low dose CsA alone or in combination with one other immunosuppressive agent for at least 12 months without a recurrence of intraocular inflammation. CsA dosage was reduced with particular attention to renal toxicity which was deemed to occur if the serum creatinine increased more than one third above the upper limit of normal values for age. ${ }^{24}{ }^{25}$ Systemic hypertension, assessed by age corrected blood pressure values, ${ }^{48}$ also necessitated CsA dosage reduction and, if appropriate, calcium antagonist therapy. ${ }^{21}{ }^{31}$ Initially, the original Sandimmun (Sandoz, Basle, Switzerland) CsA preparation was used, but when Neoral (Sandoz, Basle, Switzerland), a new microemulsion CsA formulation with better bioavailability, ${ }^{49-51}$ became available, patients were switched to Neoral on a $1: 1$ basis.

\section{Results}

Fourteen patients (25 eyes) were recruited with a mean age at first presentation with uveitis of 6.8 years (range 2.3-15.2) and at start of CsA therapy of 8.7 years (range 3.5-15.9). There were 10 males and four females with a mean follow up of 26.8 (range 3.5-88.3) months (Table 1). The most common diagnosis was pars planitis (five patients), followed by idiopathic uveitis (four patients), juvenile chronic arthritis (JCA) (three patients), and sympathetic ophthalmia (two patients). Anatomical classifications included intermediate uveitis (eight patients, 57\%), followed by panuveitis in three patients $(21 \%)$ and posterior uveitis alone in three patients (21\%). Although three patients had unilateral disease, no patient received periocular steroid injections for inflammatory recurrences. The indications for systemic CsA therapy are outlined in Table 2 with the most common indications being the failure of systemic steroids to control intraocular inflammation (six patients) and a requirement for long term steroid sparing therapy (six patients).

Visual acuity at commencement of CsA therapy was $6 / 12$ or better in 12 eyes $(48 \%)$, $6 / 18$ to $6 / 60$ in 10 eyes $(40 \%)$, and worse than $6 / 60$ to finger counting only in three eyes (12\%). Mean duration of CsA therapy was 20.9 (range 3.5-88.3) months and, in addition, two $(14 \%)$ patients had disease remission which no longer necessitated CsA therapy at the last clinic visit. The time interval from onset of CsA therapy to remission was 24.9 and 25.0 months respectively. Intraocular inflammation was controlled in seven $(50 \%)$ patients with a combination of systemic CsA and prednisolone; four (28\%) with CsA monotherapy; two $(14 \%)$ with triple therapy of systemic CsA, prednisolone, and azathioprine; and one $(7 \%)$ with a combination of CsA, prednisolone, and methotrexate. Of the 10 patients requiring systemic steroid therapy at the last clinic visit, the prednisolone dose (mean (SD)) could be weaned down to 6.3 (3.3) $\mathrm{mg} / \mathrm{kg} /$ day. The mean cumulative dose of CsA was $2.1(2.0) \mathrm{g} / \mathrm{kg}$. The mean maintenance CsA dose was 4.0 (1.0) $\mathrm{mg} / \mathrm{kg} /$ day, although the mean maximum CsA dose was $5.2(0.9) \mathrm{mg} / \mathrm{kg} /$ day. Patients $1-5$ were initially treated with Sandimmun, and then switched to Neoral, with no adverse effects (including no rise in CsA trough or creatinine levels) or dose reductions subsequent to the change in formulation. Patients 6-14 were treated with Neoral de novo.

At the last clinic visit, visual acuity was $6 / 12$ or better in 16 eyes (64\%), 6/18 to $6 / 60$ in seven eyes $(28 \%)$ and worse than $6 / 60$ to finger counting only in two eyes $(8 \%)$. Of seven eyes with final visual acuity from $6 / 18$ to $6 / 60$, the cause was maculopathy in five eyes and, of these, amblyopia associated with early onset maculopathy probably contributed to the poor BCVA in four eyes. When the final visual acuity was worse than $6 / 60$, the cause was maculopathy in one eye with probable associated amblyopia due to early onset severe uveitis. Amblyopia associated with early onset maculopathy was deemed to occur in six eyes of five patients, who had presented at a mean age of 3.4 years (range 2.3-3.9). Figure 1 shows the BCVA at the start of CsA therapy (pre CsA) and at the last visit (post CsA). Eleven eyes (44\%) improved visual acuity by at least two lines, 12 eyes (48\%) had unchanged visual acuity, and two eyes (8\%) had disimproved visual acuity by at least two lines at the last clinic visit. Anti-inflammatory efficacy 
Table 3 Renal responses to cyclosporin A therapy

\begin{tabular}{|c|c|c|c|c|}
\hline Patient No & $\begin{array}{l}\text { Maint }{ }^{\star} C s A \\
\text { dose }(m g / k g)\end{array}$ & $\begin{array}{l}\text { Max CsA } \\
\operatorname{dose}(m g / k g)\end{array}$ & $\begin{array}{l}\text { End serum creatinine } \\
\text { ( } \mu \text { molll)/ (End \% from } \\
\text { baseline) }\end{array}$ & $\begin{array}{l}\text { Normal serum creatinine for } \\
\text { age/ (\% above upper limit) }\end{array}$ \\
\hline 1 & 3.3 & 5.0 & $53(2)$ & $30-76(0)$ \\
\hline 2 & 4.2 & 5.3 & $89(98)$ & $29-85(5)$ \\
\hline 3 & 4.8 & 5.0 & $41(-16)$ & $23-73(0)$ \\
\hline 4 & 1.4 & 5.0 & $61(3)$ & $15-93(0)$ \\
\hline 5 & 3.0 & 3.0 & 79 (119) & $25-75(5)$ \\
\hline 6 & 4.3 & 5.1 & $70(32)$ & 24-80(0) \\
\hline 7 & 5.0 & 7.5 & $25(14)$ & $23-63(0)$ \\
\hline 8 & 4.0 & 5.5 & $75(42)$ & $23-73(3)$ \\
\hline 9 & 3.6 & 6.0 & $88(24)$ & $20-98(0)$ \\
\hline 10 & 3.5 & 5.0 & 80 (13) & $27-97(0)$ \\
\hline 11 & 4.7 & 5.0 & $80(14)$ & $24-80(0)$ \\
\hline 12 & 4.7 & 5.0 & 79 (5) & $30-80(0)$ \\
\hline 13 & 4.8 & 5.1 & $78(8)$ & $30-80(0)$ \\
\hline 14 & 4.2 & 5.5 & $89(6)$ & $21-109(0)$ \\
\hline
\end{tabular}

* Maint = maintenance; Max $=$ maximum $; \mathrm{CsA}=$ cyclosporin $\mathrm{A}$.

Figures are outlined in bold if the serum creatinine at the time of last visit had risen more than $30 \%$ from baseline. blood pressure returned to normal on CsA dose reduction. One patient inadvertently received a maximum CsA dose of $7.5 \mathrm{mg} / \mathrm{kg} /$ day for a period of 1 month, but this was well tolerated with no adverse renal effects and the CsA dose was reduced to a daily maintenance of $5.0 \mathrm{mg} / \mathrm{kg}$. Table 4 outlines other minor CsA induced adverse effects. The most common were hypertrichosis in four patients $(29 \%)$ and fatigue in three patients $(21 \%)$. Hypomagnesaemia occurred in two patients (14\%), with associated gastrointestinal cramps, requiring magnesium supplementation. Two patients $(14 \%)$ required CsA dose reductions because of malaise which resolved at a lower CsA dose. No patient developed anaemia, abnormal liver function tests, hypercholesterolaemia, fasting hyperglycaemia, or hyperuricaemia. With respect to systemic steroid adverse effects, five patients $(36 \%)$ developed cushingoid facies but only two $(14 \%)$ had excess weight gain (>90th centile), and all had preserved height centiles.

\section{Discussion}

The most important finding of this study is that the data suggest that CsA therapy is effective in refractory non-infectious childhood uveitis, yet is well tolerated. Most eyes (23, $92 \%$ ) had either improved or maintained best corrected visual acuity and 19 eyes $(76 \%)$ had an improved inflammatory (BIO) score with a mean follow up of 26.8 months. No patient required discontinuation of CsA therapy due to adverse effects. Nephrotoxicity did not occur but one patient developed transient systemic hypertension which resolved after CsA dose reduction. Minor adverse effects were more common yet were surprisingly well tolerated and mild in severity. One of the most positive effects of CsA therapy was to allow steroid sparing anti-inflammatory efficacy, with four patients on CsA monotherapy alone and reduction to a mean prednisolone dose of 6.3 $\mathrm{mg} / \mathrm{kg} /$ day in the other patients, and preservation of height centiles in all patients. Most patients $(64 \%)$ were treated de novo with Neoral, which is reported to have a $20 \%$ greater bioavailability than Sandimmun, ${ }^{32}$ which may have contributed to the efficacy of low dose CsA therapy in this childhood uveitis cohort. However, no specific CsA dose reduction pattern was noted in those patients who had been previously treated with Sandimmun.

To our knowledge, this is the largest reported cohort of children on CsA therapy for refractory uveitis. The number of patients in this study, however, is still small, owing to the infrequent nature of these challenging cases, even in a tertiary referral setting. One study of 60 children with intermediate uveitis reported a positive response in three of five patients treated with "immunosuppressive agents" but no comment was made on the nature of immunosuppressive therapy. ${ }^{9}$ Tugal-Tutkun and colleagues, in a recent study of 130 patients with childhood uveitis, reported therapeutic failure in three of six patients who received CsA therapy but made no comment on CsA related adverse effects. ${ }^{1}$ Both studies favoured steroid who had received a maximum daily CsA dose of $6.0 \mathrm{mg} / \mathrm{kg}$ but serum creatinine remained within normal age corrected limits and the 
therapy, with 62 patients (44\%) in the latter study receiving systemic steroids. ${ }^{1}$ Immunosuppressive therapy was required most frequently in JCA, with all CsA therapeutic failures occurring in JCA. ${ }^{1}$ On this basis, one would expect a disproportionately higher frequency of JCA in our group with refractory childhood uveitis but this has not been seen. The most common diagnosis in our group was pars planitis (36\%), with CsA therapeutic failure occurring in only one patient, who had pars planitis. A recent Italian study ${ }^{29}$ on 16 children with Behçet's disease, found that $56.3 \%$ cases required immunosuppressive therapy, with similar indications to our group, such as uncontrolled intraocular inflammation on high dose steroid therapy or the development of steroid related side effects. Cyclosporin A was used in only four cases, however, and their approach was to delay immunosuppressive therapy (mean age at commencement was 15.7 years compared with 8.4 years in our study) for as long as possible so that treatment outcomes were assessed in a much older paediatric cohort. If sight threatening intraocular inflammation is present, we recommend prompt institution of adequate systemic immunosuppression, even in very young children where the potential for amblyopia associated with aggressive uveitis is great. Similar to a previous report, ${ }^{1}$ the most common cause of reduced visual acuity was maculopathy in our group, with amblyopia probably contributing in most eyes (six of seven) with maculopathy, compared with only two of 18 eyes with maculopathy previously reported. In common with Pivetti-Pezzi and colleagues, ${ }^{29}$ we regard CsA as the immunosuppressive agent of choice, compared with potentially more toxic immunosuppressive agents such as alkylating agents or antimetabolites, in refractory paediatric uveitis.

Our approach in paediatric sight threatening intraocular inflammation is to institute short term high dose oral steroid therapy initially, to control active inflammation, and monitor the clinical response, with appropriate reductions in steroid dosage over 4-6 weeks. Depending on the adequacy of inflammatory control, a decision is made on the requirement for immunosuppressive therapy and CsA therapy is instituted (as discussed in Methods). Systemic steroid dose reduction can then be achieved, without compromising inflammatory control, and in some cases systemic steroid therapy can be eliminated, as seen in four patients in our cohort. While the majority of patients in this study still required steroids or other immunosuppressive agents, the aim of this study was not to assess CsA monotherapy alone, but to show that CsA, alone or in low dose combination therapy, was effective and safe in childhood uveitis. In particular, the addition of CsA allowed the dose reduction or elimination of other, potentially more toxic, immunosuppressive agents.

Although $50 \%$ of the patients required initial CsA dose reduction, by careful monitoring, patients avoided CsA major adverse effects (apart from one patient with transient systemic hypertension) and one of the major adverse effects of chronic systemic steroids in children, growth retardation. Cyclosporin A is being increasingly used other branches of paediatric medicine as a steroid sparing immunosuppressive agent ${ }^{17183036-39}$ but, as in this study, the long term effects, particularly any possible increased risk of neoplasia, are relatively unknown and these should be considered before commencing CsA therapy. One study of 32 children $^{18}$ found CsA therapy after renal transplantation to be very effective, over a mean of 6.5 years, and while the CsA doses were higher at $5-7 \mathrm{mg} / \mathrm{kg} /$ day, there were no malignancies. While Lane et al did not show an increased risk of malignancy in patients with severe ocular inflammatory disease treated with systemic immunosuppression, ${ }^{26}$ their cyclosporin treated group had a mean duration of therapy of only 15.4 months, with a median follow up of 1.34 years, precluding any conclusions about the long term risks of neoplasia.

Previous reports have favoured the use of periocular steroids for intraocular inflammatory recurrences in an effort to reduce systemic side effects. ${ }^{9}{ }^{1146}$ Giles reported the use of periocular steroids in $52(87 \%)$ children with intermediate uveitis, ${ }^{9}$ many requiring general anaesthesia for administration, while a review of 315 children with uveitis, mostly with JCA, found periocular steroids of considerable value in refractory cases. ${ }^{11}$ It has been recently shown, however, that periocular steroids have significant systemic absorption, with a periocular injection of $5 \mathrm{mg}$ dexamethasone achieving serum levels equivalent to $50 \mathrm{mg}$ oral prednisolone. ${ }^{52}$ While no patient received periocular steroids for inflammatory recurrences in our group, as the risk of systemic adverse effects is not avoided and systemic steroids are easier to administer with a more titratable dosage, periocular steroids may be useful in inflammatory recurrences that are unilateral or predominantly anterior. ${ }^{11} 46$

A potential criticism of this study is that only $44 \%$ eyes had a significant improvement in BCVA, but this was accompanied by $48 \%$ eyes preserving the pre CsA BCVA, which is itself an important therapeutic goal in refractory endogenous posterior uveitis (EPU), and amblyopia must also be considered as a cause of irreversible acuity loss in childhood uveitis. A potential limitation of the acuity measurements is that different methods of acuity assessment were used, particularly in the three patients with uveitis onset before the age of 5 years with more than 2 years of follow up, but this is relatively unavoidable in longitudinal childhood acuity recordings. The degree of intraocular inflammation can be reliably assessed by the BIO score ${ }^{4253}$ and is widely accepted as a reliable method of determining treatment efficacy in EPU. ${ }^{47}{ }^{54}$ Although BIO scores were reviewed retrospectively, and so may be relatively subjective, reproducibility was enhanced by the same experienced observer assessing the $\mathrm{BIO}$ score at each visit.

In conclusion, CsA is effective in refractory non-infectious childhood uveitis and relatively safe in low doses, if closely monitored, when 
compared with traditional chronic steroid therapy or potentially more toxic alternative immunosuppressive agents. The alternatives to systemic immunosuppression, however, are the immediate and long term consequences of persistent sight threatening intraocular inflammation, often with adverse effects of chronic steroid therapy, in childhood. Medium term inflammatory control may be all that is necessary in some children, as seen in this study, until disease remission takes place.

Presented as a poster at the Oxford Ophthalmological Congress, Oxford, UK, July 1997.

1 Tugal-Tutkun I, Havrlikova K, Power WJ, et al. Changing patterns in uveitis of childhood. Ophthalmology 1996; 103:375-83.

2 Perkins ES. Patterns of uveitis in children. $\mathrm{Br} \mathcal{F}$ Ophthalmol 1966;50:169-85.

3 Davis MD. Endogenous uveitis in children; associated band shaped keratopathy and rheumatoid arthritis. Arch Ophthalmol 1953;50:443-54

4 Kimura SJ, Hogan MJ, Thygeson P. Uveitis in children. Arch Ophthalmol 1954;51:80-8.

5 Kimura SJ, Hogan MJ. Uveitis in children: analysis of 274 cases. Trans Am Ophthalmol Soc 1964;62:173-92.

6 Giles CL. Uveitis in childhood. Part I Anterior. Ann Ophthalmol 1989;21:13-19.

7 Giles CL. Uveitis in childhood. Part II Intermediate. Ann Ophthalmol 1989;21:20-2.

8 Giles CL. Uveitis in childhood. Part III Posterior. Ann Ophthalmol 1989;21:23-8.

9 Giles CL. Pediatric intermediate uveitis. 7 Pediatr Ophthalmol Strabismus 1989;26:136-9.

10 Kanski JJ, Shun-Shin GA. Systemic uveitis syndromes in childhood: an analysis of 340 cases. Ophthalmology 1984;91:1247-5

11 Kanski JJ. Juvenile arthritis and uveitis. Surv Ophthalmol 1990;34:253-67.

12 Tamesis RR, Rodriguez A, Christen WG, et al. Systemic drug toxicity trends in immunosuppressive therapy of immune and inflammatory ocular disease. Ophthalmology 1996;103:768-75.

13 Santamaria J II. Steroidal agents: their systemic and ocular complications. Ocular Inflammation and Therapeutics 1983; 1:19-26.

14 Chamberlin P, Meyer WJ III. Management of pituitaryadrenal suppression secondary to corticosteroid therapy. Pediatrics 1981;67:245-51.

15 Hokken-Koelega ACS, Stijnen T, De Muinck KeizerSchrama SMPF, et al. Levels of growth hormone, IGF-I and -II, IGFBP-I and -3 and cortisol in prednisone treated and -II, IGFBP-I and -3 and cortisol in prednisone treated children with growth retardation after renal
tion. 7 Clin Endocrinol Metab 1993;77:932-8.

16 Allen DB, Goldberg BD. Stimulation of collagen synthesis and linear growth by growth hormone in glucocorticoidtreated children. Pediatrics 1992;89:416-21.

17 Hokken-Koelega AC, Van Zaal MA, de Ridder MA, et al. Growth after renal transplantation in prepubertal children impact of various treatment modalities. Pediatr Res 1994;35:367-71.

18 Brodehl J, Bokenkamp A, Hoyer PF, et al. Long-term results of cyclosporin A therapy in children. 7 Am Soc Nephrol 1992;2:S246-54

19 Nussenblatt RB, Palestine AG, Chan CC. Cyclosporin A therapy in the treatment of intraocular inflammatory disease resistant to systemic corticosteroids and cytotoxic agents. Am f Ophthalmol 1983;96:275-82.

20 Towler HMA, Cliffe AM, Whiting PH, et al. Low dose cyclosporin A therapy in chronic posterior uveitis. Eye

21 Towler HMA, Whiting PH, Forrester JV. Combination low dose cyclosporin $\mathrm{A}$ and steroid therapy in chroinic intraocular inflammation. Eye 1990;4:514-20.

22 Hemady R, Tauber J, Foster CS. Immunosuppressive drugs in immune and inflammatory ocular disease. Surv Ophthal mol 1991;35:369-85.

23 Nussenblatt RB, Palestine AG, Chan CC, et al. Randomized, double-masked study of cyclosporine compared to prednisolone in the treatment of endogenous uveitis. $A m^{f}$ Ophthalmol 1991;112:138-46.

24 Vitale AT, Rodriguez A, Foster CS. Low-dose cyclosporin A therapy in treating chronic noninfectious uveitis. Ophthalmology 1996;103:365-74.

25 Schwartz GJ, Haycock GB, Spitzer A. Plasma creatinine and urea concentration in children. Normal values for age and sex. f Pediatr 1976;88:828-30.

26 Lane L, Tamesis R, Rodriguez A, et al. Systemic immunosuppressive therapy and the occurrence of malig- nancy in patients with ocular inflammatory disease. Ophthalmology 1995;102:1530-5.

27 Feutren G, Mihatsch M. Risk factors for cyclosporineinduced nephropathy in patients with autoimmune diseases. N Engl F Med 1992;326:1654-60.

28 Nussenblatt RB, Whitcup SM, Palestine AG. Intermediate uveitis. In: Nussenblatt RB, Whitcup SM, Palestine AG, eds. Uveitis: fundamentals and clinical practice. 2nd ed. St Louis: Mosby, 1996:279-88.

29 Pivetti-Pezzi P, Accorintini M, Abdulaziz MA, et al. Behçet's disease in children. Fpn f Ophthalmol 1995;39:309-14.

30 Hoyer PF, Offner G, Wonigeit $\mathrm{K}$, et al. Dosage of cyclosporin A in children with renal transplants. Clin Nephrol 1984:22:68-71.

31 Tugwell P. International consensus recommendations on cyclosporin use in rheumatoid arthritis. Drugs 1995;50:4856.

32 Dijkmans BAC. Safety aspects of cyclosporin in rheumatoid arthritis. Drugs 1995;50:41-7.

33 International Kidney Biopsy Registry of Cyclosporin in Autoimmune Diseases. Renal morphology after cyclosporin A therapy in rheumatoid arthritis patients. $\mathrm{Br} \mathcal{7}$ Rheumatol 1993;32:65-71.

34 Tarantino A, Mantagnino G, Ponticelli C. Corticosteroids in kidney transplant patients. Safety issues and timing of discontinuation. Drug Safety 1995;13:145-56.

35 Burren CP, Jones CL, Francis DM, et al. Renal transplantation in young children. Austr NZ F Med 1995;25:122-6.

36 Chao SM, Jones CL, Powell HR, et al. Triple immunosuppression with subsequent prednisolone withdrawal: 6 years experience in paediatric renal allograft recipients. Pediatr Nephrol 1994;8:62-9.

$37 \mathrm{Au}$ J, Gregory JW, Colquhoun IW, et al. Paediatric cardiac transplantation with steroid-sparing maintenance immunosuppression. Arch Dis Child 1992;67:1262-6.

38 Neuhas TJ, Burger HR, Klinger M, et al. Long-term low-dose cyclosporin A in steroid dependent nephrotic syndrome of childhood. Eur $\mathcal{F}$ Pediatr 1992;151:775-8.

39 Hulton SA, Neuhas TJ, Dillon MJ, et al. Long-term cyclosporin A treatment of minimal-change nephrotic syndrome of childhood. Pediatr Nephrol 1994;8:401-3.

40 Assan R, Timsit J, Feutren G, et al. The kidney in cyclosporin A-treated diabetic patients: a long-term clinicopathological study. Clin Nephrol 1994;41:41-9.

41 Bloch-Michel E, Nussenblatt RB. International Uveitis Study Group recommendations for the evaluation of intraocular inflammatory disease. Am f Ophthalmol 1987; 103:234-5.

42 Nussenblatt RB, Palestine AG, Chan CC, et al. Standardization of vitreal inflammatory activity in intermediate and posterior uveitis. Am f Ophthalmol 1985;92:467-71.

43 Tanner JM, Whitehouse RH, Takaishi M. Standards from birth to maturity for height, weight, height velocity and weight velocity: British children. Arch Dis Child 1966;41: 613-35.

44 Dick AD, Towler HMA, Whiting P, et al. Assessment of renal function in cyclosporin A treatment of chronic intraocular inflammation. In: Dernouchamps JP, Verougstraete C, Caspers-Velu L, Tassignon MJ, eds. Recent advances in uveitis, proceedings of the third international symposium on uveitis, Brussels, Belgium, May 24-27, 1992. Amsterdam: Kugler Publications, 1993:521-4.

45 Whitcup SM, Salvo EC, Nussenblatt RB. Combined cyclosporine and corticosteroid therapy for sightthreatening uveitis in Behçet's disease. Am f Ophthalmol 1994;118:39-45.

46 Nussenblatt RB, Whitcup SM, Palestine AG. Philosophy, goals, and approaches to medical therapy. In: Nussenblatt RBals, Whitcup SM, Palestine AG, eds. Uveitis: fundamentals and clinical practice. 2 nd ed. St Louis: Mosby, 1996:97-134. 47 Dick AD, Azim MA, Forrester JV. Immunosuppressive therapy for chronic uveitis: optimising therapy with steroids and cyclosporin A. Br F Ophthalmol 1997;81:1107-13.

48 Hull D, Johnston DI. Heart. In: Hull D, Johnston DI, eds. Essential paediatrics. 2nd ed. Edinburgh: Churchill Livingstone, 1987:124-46.

49 Bokenkamp A, Offner G, Hoyer PF, et al. Improved absorption of cyclosporin A from a new microemulsion formulation: implications for dosage and monitoring. Pediatr Nephrol 1995;9:196-8.

50 Krmar RT, Wuhl E, Ding R, et al. Pharmacokinetics of a new microemulsion formulation of cyclosporin A (Neoral) in young patients after renal transplantation. Transpl Int 1996;9:476-80

51 Somerville MF, Scott DG. Neoral-new cyclosporin for old? Br F Rheumatol 1997;36;1113-15.

52 Weitjens O, van der Sluijs FA, Schoemaker RC, et al. Peribulbar corticosteroid injection: vitreal and serum concentration after dexamethasone disodium phosphate injection. Am f Ophthalmol 1997;123:358-64.

53 BenEzra D, Forrester JV, Nussenblatt RB, et al. Uveitis scoring system. Berlin: Springer-Verlag, 1992:1-13.

54 Ishioka M, Ohno S, Nakamura S, et al. FK506 treatment of noninfectious uveitis. Am 7 Ophthalmol 1994:118:723-9. 